\title{
Angina de Ludwig em paciente portador de esquizofrenia: relato de caso
}

\author{
Ludwig's angina in a bearer of schizophrenia patient: case report
}

\author{
Elias Almeida dos Santos ${ }^{1}$, Alana Del'Arco Barboza², César Feitoza Bassi Costa², Paloma Heine Quintas², \\ Fátima Karoline Araújo Alves Dultra ${ }^{3 *}$
}

\begin{abstract}
${ }^{1}$ Graduando em Odontologia pela Universidade Federal da Bahia - UFBA.; ${ }^{2}$ Residente do Serviço de Cirurgia e Traumatologia Bucomaxilofacial - UFBA.; ${ }^{3}$ Doutora em Processos Interativos de Órgãos e Sistemas pelo Instituto de
\end{abstract} Ciências da Saúde da Universidade Federal da Bahia - ICS/UFBA

\begin{abstract}
Resumo
Introdução: Angina de Ludwig é um processo de celulite severa, quase sempre de origem odontogênica, que envolve bilateralmente os espaços perimandibulares. Objetivo: discutir o manejo da Angina de Ludwig em paciente comprometido sistemicamente em uma situação de urgência. Relato de caso: paciente L.H.S.A., 36 anos, portador de esquizofrenia, apresentou-se à emergência do Hospital Geral do Estado cursando com disfagia, dispneia e aumento de volume endurecido e aquecido à palpação em região submandibular bilateral, sublingual e submentoniana. Foi iniciado regime de antibioticoterapia venosa e encaminhamento ao centro cirúrgico para remoção do foco causal, incisão e dissecação romba profunda, bem como inserção de drenos rígidos sob anestesia geral. Resultados: aproximadamente um mês após a intervenção inicial, o paciente apresentou remissão da infecção e segue sem recidivas. Conclusão: a suspeita clínica de Angina de Ludwig deve ser aumentada em pacientes psiquiátricos e usuários de drogas psicotrópicas, uma vez que essas condições aumentam a suceptibilidade à infecção. A preservação das vias aéreas, antibioticoterapia e o tratamento cirúrgico são eficazes na erradicação do quadro.
\end{abstract}

Palavras-chave: Angina de Ludwig. Esquizofrenia. Celulite

\begin{abstract}
Introduction: Ludwig's angina is a process of severe cellulitis, usually of odontogenic origin, which involves bilaterally the perimandibular spaces. Objective: to discuss the treatment of Ludwig's angina in a systemically compromised patient in an emergency. Case report: patient L.H.S.A., 36 years old, carrier of schizophrenia, reported to the emergency of the State General Hospital presenting dysphagia, dyspnea, and increased volume of hardened and warm palpation in the submandibular bilateral, sublingual and submental regions. The team started a venous antibiotic therapy regimen and sent the patient to the surgical center for removal of the causal focus, incision and deep blunt dissection, as well as insertion of rigid drains under general anesthesia. Results: about a month after the initial intervention, the patient presented remission of the infection and follows without relapses. Conclusion: the clinical suspicion of Ludwig's angina should be increased in psychiatric patients and users of psychotropic drugs, since these conditions increase the susceptibility to infection. Airway preservation, antibiotic therapy and surgical treatment are effective in eradicating the disease. Keywords: Ludwig's Angina. Schizophrenia. Cellulitis.
\end{abstract}

\section{INTRODUÇÃO}

A Angina de Ludwig (AL) designa um processo severo e progressivo de celulite que acomete bilateralmente os espaços perimandibulares. Este é um quadro que caracteriza emergência médica e requer tratamento imediato ${ }^{1}$. As infecções odontogênicas são as principais causas da $\mathrm{AL}^{2}$. Entretanto, outras origens têm sido relatadas, como fratura de mandíbula, abcessos peritonsilares, lesão do assoalho bucal, sialodenite, rânula infectada e linfadenite. ${ }^{3}$ A etiologia da infecção é polimicrobiana, oriunda da microbiota oral, desta forma, organismos aeróbicos e anaeróbicos são usualmente encontrados ${ }^{2,3}$. Por vezes, 0 indivíduo acometido é portador de Diabetes melitus, vírus

Correspondente/ Correspondent: * Fátima Karoline Araújo Alves Dultra - Instituto de Ciências da Saúde - UFBA - Endereço: Avenida Reitor Miguel Calmon, S/N, Canela Salvador-BA CEP: 40213-300 - Telefone: (71) 98872-7841 -E-mail: fatimadultra@gmail.com
HIV ou apresenta outro comprometimento sistêmico ${ }^{3}$.

Clinicamente, a AL comporta-se como um aumento de volume na região de pescoço e mento. A região afetada é endurecida à palpação, não-flutuante e muito sensível ${ }^{2,4}$. Além disso, o aumento de volume intraoral é responsável por causar elevação da língua. Ainda, é comum que o paciente apresente sialorreia, disfonia, odinofagia, dispneia, taquipneia, febre, dor ${ }^{4}$. A progressão do quadro pode determinar complicações fatais como obstrução das vias aéreas, mediastinite e sepse ${ }^{3}$.

O diagnóstico da AL é clínico, porém exames de imagem como tomografia computadorizada, ultrassom e ressonância magnética podem ser usados para detectar a extensão cervical da celulite ${ }^{4,5}$. Adicionalmente, os exames laboratoriais detectam aumento da contagem de leucócitos $^{2,6}$. Os critérios clínicos observados por Grodznky em 1939 ainda hoje são citados como diretrizes diagnósticas da $\mathrm{AL}^{3}$. Esse autor sugeriu que deve haver uma celulite 
perimandibular bilateral envolvendo mais de um espaço fascial; uma infiltração serosanguinolenta com pouca ou nenhuma formação de pus; uma infiltração que atinge tecido conjuntivo, fáscias e músculos, sem envolver tecido glandular; e espalha-se por continuidade dos planos teciduais e não por via linfática ${ }^{4}$.

O tratamento do quadro de AL consiste na estabilização das vias aéreas, antibioticoterapia e hidratação, remoção do foco infeccioso, incisão e drenagem. ${ }^{2,4} A$ via aérea pode ser mantida por meio de intubação nasotraqueal, orotraqueal ou traqueostomia e deve ser planejada com antecedência a fim de evitar obstrução da via aérea pelo edema parafaríngeo ${ }^{5}$. O regime antibiótico deve ser iniciado ainda na sala de emergência, contemplando drogas de amplo espectro ${ }^{4}$. Por fim, a abordagem cirúrgica envolve a descompressão dos espaços acometidos através da drenagem ${ }^{1}$.

A AL é um quadro de infecção que representa um grave risco à vida e exige preparo do cirurgião para diagnóstico precoce e manejo imediato ${ }^{1,5}$. Com este relato, espera-se contribuir com o reconhecimento da AL e com a decisão terapêutica em casos de urgência. Este trabalho visa relatar um caso de Angina de Ludwig que evoluiu após tratamento endodôntico em paciente psiquiátrico.

\section{RELATO DE CASO}

Paciente L.H.S.A., sexo masculino, 36 anos, apresentou-se à emergência do Hospital Geral do Estado, cursando com aumento de volume em região de mandíbula, referindo evolução de quatro dias. Além disso, paciente queixava-se de dispneia e odinofagia, e apresentava histórico de tratamento endodôntico na unidade 4.7 há dez dias. (Figura 1A)

Figura 1 - Apresentação clínica inicial do paciente L.H.S.A.: A: vista frontal; B: vista lateral.

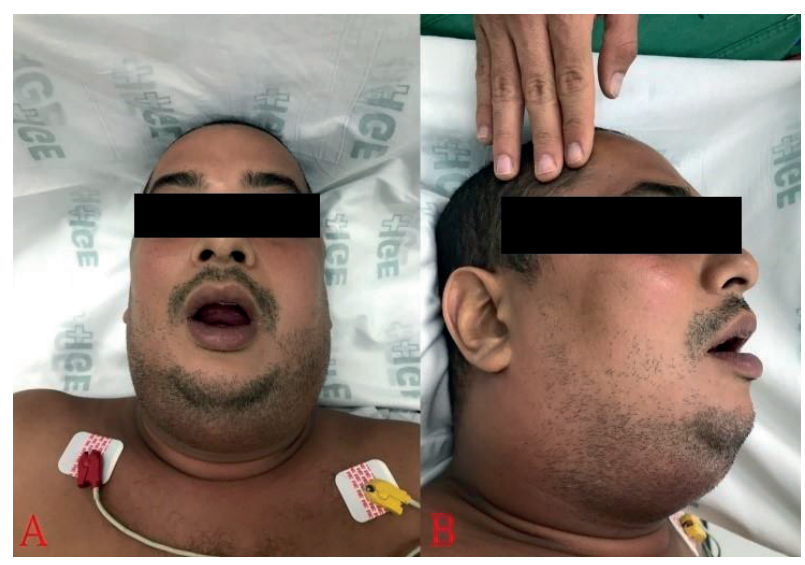

Fonte: Autoria própria

Durante anamnese, responsável refere que o paciente possui diagnóstico de esquizofrenia, em uso de Clozarpina $100 \mathrm{mg}$, Valproato de Sódio 500mg, Cloridrato de Tioridazina, Sulpirida 50mg, Cloridrato de Sertralina 100mg,
Periciazina 40mg, Tartarato de Metoprolol. Responsável nega qualquer alergia medicamentosa pelo paciente.

Ao exame físico maxilofacial, notou-se aumento de volume com limites difusos, coloração avermelhada, aquecido e endurecido à palpação em região submandibular bilateral, submentoniana e sublingual. Ao exame intraoral, observou-se elevação e retroposicionamento lingual, incapacidade de oclusão e limitação da abertura bucal. Ainda, a unidade dentária 4.7 apresentava restauração na superfície oclusal e a unidade 4.8 portava lesão cariosa na mesma superfície (Figura 1).

O exame de tomografia computadorizada detectou a densificação das partes moles dos espaços mastigatórios, porém não observou-se formação de coleções purulentas. No período da admissão, a Proteína C Reativa apresentava concentração maior que $9,0 \mathrm{mg} / \mathrm{dl}$ e o leucograma evidenciou contagem elevada de Leucócitos totais, de $19,88 \mathrm{mil} / \mathrm{mm}^{3}$. A partir dos achados clínicos, definiu-se o diagnóstico de Angina de Ludwig e optou-se pelo manejo cirúrgico do caso. Foi iniciado o regime de antibioticoterapia venosa e encaminhamento ao centro cirúrgico.

O paciente foi abordado em decúbito dorsal, com intubação orotraqueal auxiliada por Bougie, sob anestesia geral. Foi realizada assepsia e antissepsia intra e extraoral com clorexidina, aposição de campos operatórios e instalação de tampão orofaríngeo. A incisão cirúrgica foi localizada em região submandibular bilateral e submentual, seguida pela divulsão romba conectando os espaços fasciais, irrigação copiosa com soro fisiológico 0,9\% e fixação de dreno rígido com nylon 3-0 (Figura 2A e B). Ainda, foi realizada a remoção do foco infeccioso pela exodontia das unidades 4.7 e 4.8 (Figura 2C). Seguinte às suturas, um curativo compressivo foi posicionado.

Após o ato cirúrgico, o paciente foi encaminhado para Unidade de Terapia Intensiva, sendo submetido à traqueostomia. No período pós-operatório imediato, foi mantido o regime antibiótico de Clindamicina 600mg, IV, a cada seis horas, e Ceftriaxona 01g, IV, a cada doze horas. A equipe de enfermagem foi orientada a realizar diariamente higiene oral rigorosa e utilização de compressas em face protegida. Ao quinto dia pós-operatório, o dreno rígido foi removido.

No acompanhamento diário dos índices leucocitários do paciente, constatou-se uma leucocitose de 33,83 $\mathrm{mil} / \mathrm{mm}^{3}$ no terceiro dia pós-operatório, na unidade de terapia intensiva. Optou-se pela troca do regime antibiótico ainda na UTI para Cefepima 01g associado ao Metronidazol 400mg, a cada doze horas, o que resultou em regressão gradual da leucocitose, durante os cinco dias posteriores, até níveis aceitáveis. No décimo quarto dia pós-operatório, o paciente recebeu alta da UTI, sendo liberado do hospital no vigésimo sétimo dia.

Com vinte e sete dias pós-operatórios, o paciente apresenta remissão total da condição infecciosa, exibindo restauração das funções mastigatória, fonética e respiratória, bem como ótimo aspecto cicatricial. O paciente seguiu em acompanhamento por três meses (Figura 2D). 
Figura 2 - Manejo cirúrgico do paciente L.H.S.A sob anestesia geral: A: fixação do tubo orotraqueal; B: Drenos rígidos posicionados após ordenha dos espaços submandibulares; C: Ferida cirúrgica pós exodontia das unidades 4.6 e 4.7. D: Pós-operatório de 03 meses

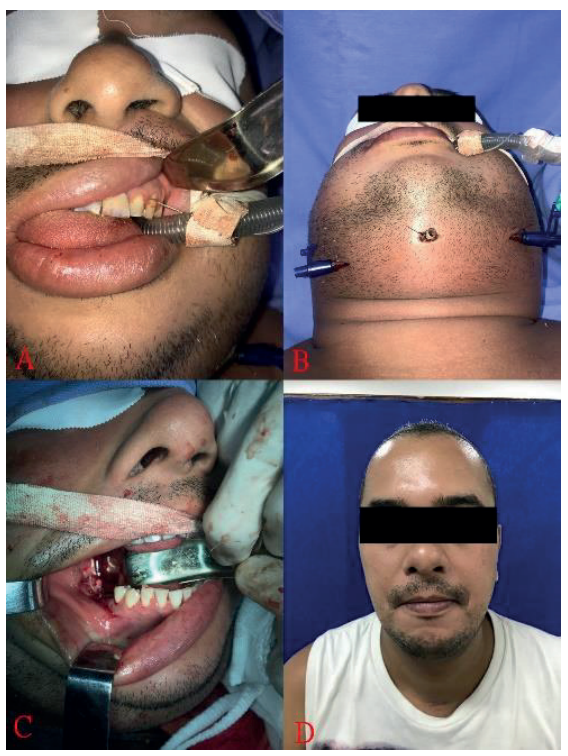

Fonte: Autoria própria

\section{DISCUSSÃO}

As celulites cérvico-faciais representam infecções difusas do tecido subcutâneo em planos teciduais da cabeça e pescoço ${ }^{7}$. Karl Friedrich Wilhelm von Ludwig foi quem primeiro discriminou um processo rapidamente progressivo de celulite, envolvendo os tecidos do assoalho bucal e laringe, a Angina de Ludwig $(A L)^{3}$. Este quadro apresenta prevalência em indivíduos do sexo masculino entre vinte e sessenta anos de idade ${ }^{3,4}$. As infeções odontogênicas, correspondem a $70 \%$ das causas de $A L$, sendo os 2 ㅇ e 3 응 molares da mandíbula os principais focos da infecção ${ }^{2,4}$. Esses dados corroboram com o perfil do paciente relatado neste caso e justificam a remoção das unidades 4.7 e 4.8 durante o manejo cirúrgico. As raízes destes molares permitem acesso direto da infecção odontogênica ao espaço submandibular, viabilizando a expansão do processo por contiguidade ao espaço sublingual e espaços profundos do pescoço ${ }^{8}$.

O quadro clínico do presente caso é compatível com o descrito na literatura sobre $A L^{1,4}$. O paciente apresentava tumefação endurecida em região perimandibular, disfonia, odinofagia, dispneia, elevação e deslocamento da língua. O deslocamento superior e posterior da base lingual é responsável por uma das mais frequentes complicações da $A L$, a obstrução de via aérea ${ }^{1,8}$. Outras complicações, como mediastinite, epiema torácico, osteomielite ou faciíte necrosante, não foram observadas neste caso. A leucocitose é, também, um achado usual nos pacientes com $\mathrm{AL}^{9}$. Contagens de leucócitos entre 7.300/ $\mu \mathrm{L}$ e $26.000 / \mu \mathrm{l}$ foram relatadas ${ }^{2,6}$. Nesse caso, o paciente apresentou-se inicialmente com valores de leucócitos 19,88 mil/ $\mathrm{mm}^{3}$ (Referência: 4,0 a 10,0 mil/ mil/ $\mathrm{mm}^{3}$ ), com expressivo aumento da fração de segmentados. Além disso, os níveis da PCR estavam aumentados, constatando a presença da infecção.

Desde o advento dos antibióticos, a taxa de mortalidade dos casos de AL foi reduzida de $50 \%$ a $8 \%$ 4,8. Entretanto, os números de óbitos permanecem proeminentes na presença de complicações, como a Faciíte Necrosante Cérvico-facial ${ }^{6}$. Em estudo com 77 casos de infecções profundas do pescoço, pesquisadores observaram que a Angina de Ludwig correspondeu a $18 \%$ dos casos, sendo a segunda localização mais comum deste tipo de infecção ${ }^{9}$. Ainda, os casos de Ludwig foram correlacionados com alto risco de complicações e maior tempo de internamento, além de representarem 100\% dos óbitos do mesmo estudo ${ }^{9}$. Esses dados ratificam a gravidade da AL e a necessidade de diagnóstico precoce e tratamento apropriado. A apresentação tardia do paciente e o atraso na abordagem terapêutica têm sido propostos como fatores para o aumento das fatalidades ${ }^{3}$. Nosso paciente evoluiu com a celulite aproximadamente 10 dias antes de apresentar-se à emergência, o que pode ter contribuído para um quadro tão avançado.

Para o nosso conhecimento, não há relatos na literatura que associe a condição mental do paciente relatado ao desenvolvimento da $\mathrm{AL}^{10}$. Entretanto, sabe-se que indivíduos com desordens psiquiátricas tendem a apresentar hábitos de higiene oral deficientes ${ }^{11}$. Além disso, tais pacientes são usuários constantes de drogas psicotrópicas ${ }^{10}$. Em estudo de revisão, foi demonstrado que existe uma associação entre o uso drogas antipsicóticas, antidepressivas estabilizantes de humor, $\beta$-bloqueadores e inibidores da receptação de serotonina com o desenvolvimento de xerostomia, maior prevalência de cárie e maior risco ao acometimento de infecções orais ${ }^{11}$. O paciente do presente relato é usuário crônico de sete medicamentos pertencentes às classes citadas neste estudo. Ademais, as doenças psiquiátricas representaram $10 \%$ das comorbidades presentes em uma série de cento e seis casos de AL publicados em $2009^{10}$. Os autores relacionaram este achado aos efeitos colaterais das drogas antidepressivas aliados à propensão ao consumo de tabaco e pior qualidade de higiene oral dos pacientes psiquiátricos.

O paciente deste caso foi submetido ao exame de tomografia computadorizada com o intuito de avaliar a extensão cervical da infecção, bem como avaliar presença de abcessos. Não foram reveladas coleções purulentas e o processo infeccioso demonstrou-se limitado acima do osso hioide. Os exames imagiológicos executam papéis essenciais nas infecções profundas do pescoço; entre eles a confirmação do diagnóstico, a diferenciação entre celulite e abcesso e o monitoramento da extensão cervical da infecção. O exame de tomografia computadorizada é vantajoso por associar rápido tempo de execução à análise anatômica precisa ${ }^{9,12}$. Alguns autores recomen- 
dam a ultrassonografia como exame de escolha para análise das vias aéreas por sua portabilidade, indolência e baixo custo. Além disso, os mesmos autores salientam que a análise das vias aéreas e estimação do diâmetro glótico devem ser solicitados independente do exame de escolha, objetivando a melhor tomada de decisão no manejo das vias aéreas ${ }^{5}$. Em contraste, a não realização de exames de imagem em casos de AL têm sido sugerida em alguns casos, a fim de otimizar o tempo de tratamento emergencial ${ }^{1}$.

A estabilização das vias aéreas é o primeiro passo no tratamento da $\mathrm{AL}^{3,5}$. Diversas técnicas estão disponíveis para este manejo, incluindo a intubação às cegas, intubação guiada por fibra óptica ou por GlideScope, e a traqueostomia ${ }^{3}$. Enquanto alguns autores consideram a traqueostomia sob anestesia local uma técnica segura, ${ }^{2,8}$ este procedimento tem sido relacionado com a possibilidade de espalhamento da infecção em direção ao peito, desenvolvimento de estenose traqueal e pneumotórax, além de ser de difícil execução em estágios avançados da $A L^{13}$. A intubação nasal às cegas deve ser evitada pois pode causar sangramento e laringoespasmo ${ }^{1}$. Alguns autores argumentam que a técnica de escolha depende da severidade da infecção, experiência clínica do operador e disponibilidade de equipamento ${ }^{14}$. $O$ paciente do presente caso foi submetido a intubação orotraqueal com auxílio de Bougie. Entretanto, a via aérea cirúrgica foi executada no pós-operatório, em razão da evolução iminente do edema e do tempo de internamento prolongado previsto. Recomenda-se a permanência da intubação entre 24 e 48 horas após a cirurgia para garantir a segurança da via aérea durante a regressão do edema ${ }^{1}$.

O manejo cirúrgico da AL é controverso. ${ }^{13,14}$ Embora alguns autores preconizem a incisão e drenagem com protocolo terapêutico, esta abordagem pode ser ignorada em casos seletos ${ }^{13}$. Em uma série de trinta e nove casos de AL publicada em 2007, os autores trataram $69 \%$ dos quadros apenas com observação das vias aéreas e administração endovenosa de antibióticos ${ }^{13}$. Por outro lado, outros autores observaram que os casos tratados através de descompressão cirúrgica foram associados com menor tempo de internamento na UTI e de estadia no hospital, com média de dois e cinco dias, respectivamente ${ }^{14}$. No presente caso, o paciente foi abordado cirurgicamente após intubação orotraqueal, através de incisões, divulsão e ordenha dos espaços submandibulares bilateral e submentual. O objetivo da descompressão cirúrgica por meio da drenagem é permitir o retorno da língua para uma posição mais anterior e inferior, recuperando a via orofaríngea, bem como permitir a oxigenação dos espaços profundos com consequente dano às espécies anaeróbicas $^{14,15}$. Além disso, a antibioticoterapia por si só pode não ser tão efetiva, uma vez que coleções purulentas distantes da corrente sanguínea não são perfundidas pelas drogas, mesmo quando administradas por via parenteral ${ }^{7}$.

As investigações microbiológicas da AL revelam uma população semelhante à microbiota oral, polimicrobiana, apresentando micro-organismos aeróbios e anaeróbicos, como espécies de Estafilococos, Estreptococos, Prevotela, Fusobactéria e Preptoscocos ${ }^{3,9}$. A antibioticoterapia de largo espectro deve ser iniciada assim que possível ${ }^{4}$. Inicialmente, a prescrição pode ser empírica, porém testes de cultura e sensibilidade devem ser solicitados para terapêutica específica. A prescrição antibiótica instituída para o paciente deste relato consistiu em um regime com Metronidazol associado à Ceftriaxona. Esta conduta está em consonância com uma publicação recente que recomenda o uso de Cefalosporinas de 3 a geração e Metronidazol ${ }^{9}$. Outro estudo preconiza o uso inicial de Amoxicilina/Clavulanato e Metronidazol ${ }^{3}$. Essas drogas combinam amplo espectro de ação e boa tolerância. ${ }^{16} \mathrm{Pe}$ nicilina $\mathrm{G}$ e Clindamicina são outras drogas recomendadas 2,13,17. A variação na contagem leucocitária observada nos primeiros dias pós-operatórios podem ter decorrido da ausência de um teste de cultura e sensibilidade prévio à prescrição antibiótica, como é recomendado por alguns autores ${ }^{10,17 .} \mathrm{O}$ uso de anti-inflamatórios não esteroidais (AINES) é contraindicado para celulites cérvico-faciais por agir na inibição da resposta imunológica ${ }^{18}$. Em relato de setenta casos, foi observada uma forte relação entre o uso de AINES e a progressão da infecção nos casos de celulite. ${ }^{7}$ Curiosamente, o uso de esteroides endovenoso é recomendado com a finalidade de reduzir o edema e facilitar a intubação $0^{2,4}$.

\section{CONCLUSÃO}

A AL é uma infecção progressiva e potencialmente fatal, o diagnóstico precoce e a abordagem terapêutica apropriada são decisivos para a cura. Pacientes especiais, polifarmácia e usuários de drogas psicotrópicas devem aumentar a suspeita clínica da AL, tendo em vista a maior susceptibilidade destes indivíduos a infecções. A manutenção e vigilância das vias aéreas é uma prioridade e o tratamento cirúrgico através da remoção de foco infeccioso, incisão e drenagem aliados à antibioticoterapia são medidas terapêuticas eficazes.

\section{REFERÊNCIAS}

1. BENNANI-BAÏTI, A. A.; BENBOUZID, A.; ESSAKALLI-HOSSYNI, L. Cervicofacial cellulitis: the impact of non-steroidal anti-inflammatory drugs. A study of 70 cases. Eur. Ann Otorhinolaryngology Head Neck Dis., Amsterdam, v. 132, n. 4, p. 181-184, 2015. Disponível em: <http://dx.doi. org/10.1016/j.anorl.2015.06.004>. Acesso em: 01 Oct. 2018.

2. BOTHA, A.; JACOBS, F.; POSTMA, C. Retrospective analysis of etiology and comorbid diseases associated with Ludwig's Angina. Ann. Maxillofac. Surg., India, v. 5, n. 2, p. 168, 2015. Disponível em: <http:// www.amsjournal.com/text.asp?2015/5/2/168/175758>. Acesso em: 01 Oct. 2018].

3. CANDAMOURTY, R. et al.Ludwig's angina - An emergency: a case report with literature review. J. Nat. Sci. Biol. Med., India, v. 3, n. 2, p. 206, 2012. Disponível em: <http://www.jnsbm.org/text. asp?2012/3/2/206/101932>. Acesso em: 01 Oct. 2018.

4. CHANG, B. A. et al. Needle aspiration versus incision and drainage for the treatment of peritonsillar abscess. Cochrane Database Syst. 
Rev., Oxford, v. 12, n. Jan. 2008, p. CD006287, 23 jul. 2016. Disponível em: <http://myspot.mona.uwi.edu/wimjopen/article/51>. Acesso em: 01 Oct. 2018].

5. COSTAIN, N.; MARRIE, T. J. Ludwig's Angina. Am. J. Med., New York, v. 124, n. 2, p. 115-177, Feb. 2011. Disponivel em: http://dx.doi. org/10.1016/j.amjmed.2010.08.004>. Acesso em: 01 Oct. 2018.

6. DARAMOLA, O. O. et al. Diagnosis and treatment of deep neck space abscesses. Otolaryngol. head neck surg., Rochester, v. 141, n. 1, p. 123-130, July 2009. Disponível em: <http://dx.doi.org/10.1016/j. otohns.2009.03.033>. Acesso em: 01 Oct. 2018.

7. FRATTO, G.; MANZON, L. Use of psychotropic drugs and associated dental diseases. Int. j. psychiatry med., New York, v. 48, n. 3, p. 185197, 2014. Disponível em:<http://journals.sagepub.com/doi/10.2190/ PM.48.3.d>. Acesso em: 01 Oct. 2018.

8. GREENBERG, S. L. L. et al. Surgical management of Ludwig's angina. ANZ j. surg, Carlton, v. 77, n. 7, p. 540-543, July 2007. Disponível em: <http://www.ncbi.nlm.nih.gov/pubmed/17610689>. Acesso em: 01 Oct. 2018.

9. KASSAM, K.; MESSIHA, A.; HELIOTIS, M. Ludwig's Angina: the original angina. Case Rep. Surg., New York, v. 2013, p. 974269, 2013. Disponível em: <http://dx.doi.org/10.1155/2013/974269>. Acesso em: 01 out 2018.

10. MANASIA, A. et al. Ludwig's angina complicated by fatal cervicofascial and mediastinal necrotizing fasciitis. IDCases, [s.I], v. 4, p. 32-33, 2016. Disponível em: <http://dx.doi.org/10.1016/j.idcr.2016.03.001>. Acesso em: 01 Oct. 2018.

11. MAROLDI, R.; FARINA, D.; RAVANELLI, M. Emergency imaging assessment of deep neck space infections. Semin. ultrasound CT MR, Philadelphia, v. 33, n. 5, p. 432-442, Oct. 2012. Disponível em: <http:// dx.doi.org/10.1053/j.sult.2012.06.008>.Acesso em: 01 out 2018.
12. NARENDRA, P. L.; VISHAL, N. S.; JENKINS, B. Ludwig's angina: need for including airways and larynx in ultrasound evaluation. BMJ case reports, London, v. 2014, p. 10-13, 9 Nov. 2014. Disponível em: <http:// www.ncbi.nlm.nih.gov/pubmed/25385564>.Acesso em: 01 Oct 2018.

13. NICOT, R. et al. Do anti-inflammatory drugs worsen odontogenic cervico-facial cellulitis? Revue de Stomatologie, de Chirurgie Maxillo-faciale et de Chirurgie Orale, [s.I], v. 115, n. 5, p. 31-36, 2014. Disponível em: <http://dx.doi.org/10.1016/j.revsto.2013.07.020>. Acesso em: 01 Oct. 2018.

14. PAK, S. et al. Ludwig's Angina. Cureus, [s.I], v. 9, n. 8, p. e1588, 21 ago. 2017. Disponível em: <http://www.cureus.com/articles/8691-ludwigs-angina>.Acesso em: 01 Oct. 2018.

15. POESCHL, P. W. et al.. Endodontic pathogens causing deep neck space infections: Clinical impact of different sampling techniques and antibiotic susceptibility. J. Endod., Baltimore, v. 37, n. 9, p. 1201-1205, 2011. Disponível em: <http://dx.doi.org/10.1016/j.joen.2011.05.029>. Acesso em: 01 Oct. 2018.

16. RATH, E. et al. Aetiology and clinical features of facial cellulitis: a prospective study. J. infect. dis., Chicago, v. 50, n. 1, p. 27-34, 2018. Disponível em: <https://doi.org/10.1080/23744235.2017.1354130>. Acesso em: 01 Oct. 2018.

17. ROWE, D. P.; OLLAPALLIL, J. Does surgical decompression in Ludwig's angina decrease hospital length of stay? ANZ j. surg., Carlton, v. 81, n. 3, p. 168-171, Mar. 2011. Disponível em: <http://www.ncbi.nlm.nih.gov/ pubmed/21342390>.Acesso em: 01 Oct. 2018.

18. SAKARYA, E. U. et al. Clinical features of deep neck infection: analysis of 77 patients. Kulak Burun Bogaz Ihtisas Derg., Turkey, v. 25, n. 2, p. 102-108, 15 maio 2015. Disponível em: <http://www.kbbihtisas. org/v02/jvi.php?pdir=kbbihtisas\&plng=tur\&un=KBBI-76500\&look4=>. Acesso em: 01 out 2018.

Submetido em: $20 / 10 / 2018$

Acesso em: 22/04/2019 